\title{
Developing the Adjudicated Case Study Method
}

\section{SUSAN STEPHEN $^{a, b}$ \& ROBERT ELLIOTT ${ }^{a}$}

\author{
${ }^{\text {a }}$ University of Strathclyde \\ ${ }^{\mathrm{b}}$ Correspondence concerning this article should be addressed to Susan Stephen, Counselling Unit, School of \\ Psychological Sciences and Health, 76 Southbrae Drive, University of Strathclyde, Glasgow G13 1PP UK \\ Email: susan.stephen@hotmail.co.uk
}

\begin{abstract}
In this commentary we discuss Miller's Panel of Psychological Inquiry (PPI) and Bohart's Research Jury method approaches to the development of the adjudicated case study method, as represented by the papers assembled for this issue of Pragmatic Case Studies in Psychotherapy. In our view, the case studies presented here demonstrate the rapidly developing potential offered by this approach for psychotherapy research and reveal many parallels to recent research using the Hermeneutic Single Case Efficacy Design (HSCED) model. In our view, each of the three models has taken significant steps forward in adapting particular aspects of the legal process as viable psychotherapy research procedures. In this commentary we summarize the HSCED method, then take readers through the issues of the sources of the evidence used; ways in which that evidence is tested; claims, burden and standard of proof; and the handling of the adjudication process itself. We conclude with recommendations for further development of adjudicated case study methods.
\end{abstract}

Key words: adjudicated case study method, Panel of Psychological Inquiry, Research Jury Method, Hermeneutic Single Case Efficacy Design (HSCED), psychotherapy research; evidence; burden of proof; cross-examination; case studies; clinical case studies

\section{INTRODUCTION}

This issue of Pragmatic Case Studies in Psychotherapy marks an important stage in the development of the adjudicated case study method within psychotherapy research. Here we have the opportunity to see inside two alternative models within the approach-Miller's Panel of Psychological Inquiry (PPI; Miller, 2011) and Bohart's Research Jury method (RJ; Bohart, Tallman, Byock, \& Mackrill, 2011) —and in enough detail to be able to develop our own sense of the processes and to understand generally the experiences of the participants.

In this commentary it is our intention to add to this process by sharing our perspective of these two models through a discussion of what we see as the key themes of adjudicated case study methods within psychotherapy research. In our discussion we will also compare and contrast the responses that these two models have taken to these themes, with that taken by a third model - the Hermeneutic Single Case Efficacy Design (HSCED) method (Elliott, 2001; 2002; Elliott et al., 2009; MacLeod, Elliott \& Rodgers, in press; Stephen, Elliott \& MacLeod, 2011)—developed by the second author of this commentary. 


\section{The Hermeneutic Single Case Efficacy Design (HSCED) method}

We begin with a short introduction to the HSCED method, inspired and building on Bohart and colleagues’ (Bohart \& Boyd, 1997; Bohart \& Humphreys, 2000) early explorations of adjudicated case study methods, and developed in response to many of the same concerns described by Miller (2011) and Bohart, Tallman, et al. (2011). In particular, we are critical of the "causal emptiness" (Elliott, 2002, p.2) implicit in the design of the randomized controlled trial design, and want to develop a systematic methodology for single case studies that would minimise the traditional criticisms made of the case study as a research method: for example, reliance on anecdotal evidence, confirmatory bias and narrative smoothing (McLeod, 2010). The original aim of the HSCED method was to evaluate the efficacy of psychotherapy on a case by case basis by asking two key questions: (1) did the client change? and (2) was the therapy generally responsible for this change? While these two questions remain at the center of its design, they have been revised and augmented as the HSCED method has developed. At present, the standard questions used in an HSCED investigation (e.g., Stephen, Elliott \& MacLeod, 2011; MacLeod, Elliott \& Rodgers, in press) are:

1. Did the client change substantially over the course of therapy?

2. Is this change substantially due to the effect of the therapy?

3. What factors (including mediator and moderator variables) may be responsible for the change?

In its original form, an HSCED investigation could be carried out by a single researcher as the only participant beyond the client and therapist whose psychotherapeutic experience was the focus of the study. In fact, the original idea was that therapists could carry out HSCED studies on their own clients, as a way of bridging the research-practice gap. The method was conceived as a "practical reasoning system with the specific purpose of evaluating the causal role of therapy in bringing about outcome” (Elliott, 2002, p.3). Then, as now, there were a number of essential ingredients required for an HSCED investigation.

First, a rich case record was created that comprised some basic background information about the client and therapist, in addition to a comprehensive set of quantitative and qualitative data that represented multiple sources, measures and perspectives relating to the therapy process and outcome.

Second, the researcher analyzed the rich case record to identify direct evidence that supported an inference that a clear link existed between therapy process and outcome. Elliott (2002) proposed five types of direct evidence: (1) retrospective attribution; (2) processoutcome mapping; (3) within-therapy process-outcome correlation; (4) early change in a stable problem; and (5) event-shift associations. This process has become known as developing the "affirmative" case.

Third, the researcher sought, in good faith, to identify data in the rich case record that supported alternative explanations for any apparent client change. Elliott (2002) set out eight types of indirect evidence that might support an alternative understanding of the therapy process and outcome: (1) trivial or negative change; (2) statistical artifacts; (3) relational artifacts; (4) expectancy artifacts; (5) self-correction processes (self-help and self-generated return to baseline functioning); (6) extra-therapy events; (7) psychobiological causes; and (8) 
reactive effects of research. This process has become known as the development of the "skeptic" case.

Finally, the researcher evaluated the two analyses of the rich case record to draw a conclusion about the likelihood that the client had changed over the course of therapy and that the therapy was a key influence on this outcome.

Since Elliott (2002) presented his HSCED analysis of the case of "Paul," the method has been developed in several ways. In particular, the HSCED investigation presented by Elliott et al. (2009) introduced significant changes to the method. In this study, two research teams were convened: one team prepared the affirmative case, while the other developed the skeptic case. A new three-part structure for the presentation of the two cases was used: a brief, a rebuttal of the opposing brief, and a summary narrative. In addition, an adjudication process was introduced. Three independent "judges" were invited to evaluate the evidence presented by the affirmative and skeptic teams and to give their opinions on the central research questions of client change and the causal role of the therapy in that change. On publication, the material that comprised the HSCED investigation (the rich case record, the affirmative and skeptic cases, and the judges' opinions) were made available as online appendices to enable readers to carry out their own adjudication and thereby test the validity of the HSCED process.

Many of the developments to the HSCED method introduced by the Elliott et al. (2009) study have now become integral parts of the process. For example, it now would be unusual for an HSCED investigation not to include an adjudication panel. One aspect of the Elliott et al. (2009) study that remains less common, at present, is the use of separate research teams in the development of the affirmative and skeptic cases. The majority of recent HSCED investigations have been carried out by single researchers, often as part of masters or doctoral research projects. This is a significant practical advantage, allowing the method to be scaled up or down according to the resources available.

Even in this brief description of the HSCED process, readers will already have recognized several similarities between this method and the two models of adjudicated case studies presented within this issue of Pragmatic Case Studies in Psychotherapy. We are now ready to move on to discuss the key themes of this approach to psychotherapy research and our commentary on how these themes are handled in each of the three models.

\section{NATURE OF EVIDENCE}

\section{Source of Evidence}

In his paper presented in this issue, Miller (2011) places the HSCED method within one of two strands that he has identified as developing within the current movement to develop case studies as a formal research method. He describes the strand within which he places the HSCED as "primarily interested in integrating case study research into studies that are pre-planned to test specific clinical hypotheses" (p. 7). In contrast he places his own work, and that of Bohart and his colleagues, within a second strand in which the participants are "invested in developing a case-study methodology that systemizes and validates the clinical knowledge that grows out of clinical practice as it naturally occurs in clinical settings” (p. 7). Although we initially considered this division into strands as somewhat 
artificial — we are keen to encourage practitioners to employ a basic version of the HSCED method within clinical settings - a consequence of this apparent distinction becomes more clear to us when we consider the choice made here by both Miller (2011) and Bohart, Tallman et al. (2011) in the selection of pre-existing narrative case studies as the main source of the evidence on which to base the adjudication process.

The rich case record compiled for an HSCED investigation presents the data collected from the client and therapist with only minimal, descriptive analysis. This provides a structured format for presenting the separate voices of the client and therapist, although they are not directly participating in the HSCED process itself. On the other hand, we would argue, using a narrative case study as the source of evidence for an adjudicated case study can limit and excessively filter the presentation of direct evidence collected from the participants in the therapeutic encounter. For example, in Podetz's (2011a) case study (in the Miller project), readers hear about the process and outcome of therapy from the therapist's perspective only. In comparison, the researcher's narrative in Mackrill's (2011) case study (in the Bohart project) is supported by a significant number of excerpts from the research diaries of the client and therapist at the center of the study, reorganized thematically and in accord with the author's theoretical interests. This approach does serve to enhance the voice of the client within the case study. We also note that both client and therapist reviewed and confirmed the accuracy of the researcher's narrative from their perspectives. Nevertheless, the fact that the members of the jury in this case have access only to excerpts reduces their ability to hear and understand the client's and therapist's words within the fuller context (i.e., the diary entries) in which they were originally expressed.

In contrast, the rich case record at the heart of the HSCED method contains the full transcript of a semi-structured research interview with the client (the Change Interview; Elliott, Slatick \& Urman, 2001) in which the client is invited to describe any changes that they have experienced over the course of their therapy, the attributions that they make for these changes, any helpful and hindering aspects of their therapy process, any personal attributes that may have helped or hindered them to use the therapy process, as well as helpful and hindering aspects of, or events in their lives while they were in therapy. Thus, not only does the researcher who prepares the affirmative and skeptic cases have access to the client's perspective of their change and the factors that influenced their process, but so too do the judges who carry out the HSCED adjudication, and any readers who wish to immerse themselves in the rich case record. Furthermore, in this model, the client's voice can also be heard in the responses that they give to the quantitative outcome measures completed before, during and at the end of therapy, and in the other qualitative measures used to capture their experience of the therapy process (e.g., Helpful Aspects of Therapy; Llewelyn, 1988).

\section{Use of Witnesses}

While Bohart's Research Jury model and HSCED both make substantial attempts to include the voices of client and therapist as witnesses to the therapeutic encounter within their case study evidence, what distinguishes the Panel of Psychological Inquiry model is the panel hearing that is convened to hear the testimony of witnesses to the case under examination. Miller (2011) notes:

The central witness to a tort trial is often the injured party, and by analogy the central witness in a case study Panel of Inquiry hearing would be the client. One cannot deny the 
logic but... it is unlikely that many therapists would or should risk the potential disruption of the therapeutic relationship and harm to the client that would result from appearing before the Panel. (p. 24)

Nevertheless, Miller (2011) makes a convincing argument for accepting the therapist as a witness to the therapeutic encounter and its impact on the client: testifying to her own observations and experiences with the client; acting as an expert witness to the client's process; and also by eye witness reporting on what the client has said-reports that Miller argues would "fall under the exceptions to the hearsay rules in the Federal Rules of Evidence” (p. 14). The plausibility of this argument is fundamental to the model that Miller presents. If the therapist cannot stand as the central witness to the therapy (in the absence of the client) then the panel model cannot proceed.

From our point of view, including the supervisor as a witness played a critical part of the PPI process. Her testimony, including her report on the supervision process, presumably based as well on her access to recordings of therapy sessions, provided essential corroboration of the therapist's evidence. By doing so, it also admitted an additional perspective to the proceedings, enabling the richness provided by multiple perspectives on therapy outcome and process. At the same time, it is interesting to read therapist Podetz's (2011b) account of her experience as the therapist during the testimony of her supervisor, in which she reports how,

very difficult [it was] to listen to others discuss and critique one's performance and almost impossible to do so quietly and without interruption. It is apparent that the lack of control during this portion of the Inquiry was challenging, as I feared that my and Anna's voices would not be heard. (p. 90).

\section{TESTING THE EVIDENCE}

\section{Cross-Examination}

We argue that at the heart of the adjudicated case study approach is the requirement to test or "cross-examine" the evidence. The proposition is that if an alternative interpretation of the evidence is experienced as plausible by the judges or jurors, then the likelihood that the claim is valid must be diminished. The way in which this process is carried out within the PPI model most closely resembles that followed in a court of law because it is possible to carry out a cross-examination of the evidence by the direct questioning of witnesses under oath. This process is observed by the panel members, who can ask further questions of the witnesses if necessary.

In contrast, cross-examination of evidence within the HSCED model takes place as an analysis of the rich case record, written up and presented for the consideration of the judges who take part in the adjudication process. This more academic approach lacks the drama of the live cross-examination of witnesses; however, we argue that it retains the essential critical-reflective approach now considered to be essential principle of systematic case study research (McLeod, 2010) and carries a very similar challenge for the HSCED Skeptic as that experienced by the PPI’s Critic (DiGiorigianni, 2011). 


\section{The Role of the Critic/Skeptic}

In his paper in this issue, DiGiorgianni (2011) discusses the struggle that he experienced in performing the role of Critic. He tells us that "no role in the Panel was more difficult to establish..." and describes in detail his discomfort at the idea of making "a commitment tantamount to denying the good work and empathic skill of the therapist in this case” (p. 75). For this reason, he tells us, he chose to take a dialectical approach to the Critic's role, rather than the adversarial stance more commonly seen in the legal model, arguing that:

the rich multiplicity of meanings capable of being generated by dialectical processes is the primary desideratum of such a panel of inquiry and, moreover, that the nuance and ambiguity attendant upon such a dialectic is so central to any truly therapeutic undertaking that it is crucial we learn to live with this condition in our case studies as well as our session hours. (p. 75)

We immediately recognized the dilemma experienced by DiGiorgianni. His struggle highlights the difficulty of enacting a Critic/Skeptic role that contradicts one's training and sensibilities as a psychological helper who has sensitivity to the relational and therapeutic context of one's actions and words. In this type of case study there is an inherent risk of harm to the client or the therapist within the case when seeking to present an alternative interpretation of the evidence of what took place within or as a result of the therapeutic encounter. In our HSCED work we are highly aware of this risk and continue to grapple with it. We have developed various methods to try to deal with this problem, attempting (a) to clarify for the client, therapist and readers, the purpose of the Skeptic case, and (b) to minimize the impact of the case on the participants. First, we have introduced a "disclaimer" heading at the top of both the affirmative and skeptic case documents. In its latest iteration (MacLeod et al., in press), this disclaimer reads, "The arguments presented here are made to facilitate the analysis of change in this case through the presentation of contrasting views; they are not necessarily the personal views of the author.” Second, when writing the Skeptic case, the researcher strives to avoid the use of language that may invalidate the client's experience of the therapy; prior to distribution to judges, all skeptic documents are audited by the research supervisor to locate and modify any potentially invalidating language. Third, we continue to develop our consent process so that the client can make an informed choice to have their therapy examined in an HSCED study, and we offer support to our clients in the event that they experience distress, for any reason, as a result of their participation in the case study process. Ultimately, we would like to create processes by which therapist and client can check and correct all affirmative and skeptic documents prior to adjudication, similar to the procedure used by Mackrill (2011) in his case study write-up.

So far there have been few HSCED investigations carried out in which the therapist has not been part of the research team. Podetz's second paper (2011b) makes a very useful contribution to our understanding of the impact of the Critic/Skeptic's role on therapists and raises the question of how best to deal with it. We suspect that her experience of the process is likely to be shared by many, which makes it important to find a way to develop the adjudicated approach to case studies so that it can be experienced by therapists as an opportunity that promotes “enlightenment and growth” (Podetz, 2011b, p. 93). 
Nevertheless, in the case of Anna we agree with the two judges who found the Critic's approach "problematic” (Miller, Kessler, et al., 2011, p. 99). A “clash” between the two opposing sides, especially if it could have been done while maintaining collegiality, would have been helpful in order to determine the strength of the alternative interpretations of the evidence.

\section{Criteria for the Evaluation of Evidence}

In Bohart's Research Jury method, there is no formal process of cross-examination. Indeed, one of the fundamental differences between this method and the other two discussed here is the lack of interpretation of the evidence prior to its evaluation by the jurors themselves. Instead, the jurors are provided with a detailed list of suggested but optional criteria that they may wish to use to assist them in evaluating the evidence for their adjudication of the case study questions (Bohart, Tallman, et al., 2011, Appendix 1). Within this extensive list, there are numerous criteria for evaluating evidence that the person did or did not change, and whether it was therapy that helped or not, including evidence for a plausible narrative case linking therapy work to positive changes. These various sets of criteria overlap with the eight types of indirect evidence used by the skeptic case within the HSCED process to support an alternative understanding of the evidence. This is not surprising, because the development of the HSCED method was strongly influenced by an earlier, shorter version of this list, presented in Bohart and Boyd (1997).

Nevertheless, in the Research Jury model, it becomes the responsibility of the jurors to ensure that they carry out the robust testing of the evidence that is so necessary for this process. This stance is encouraged in the Instructions to Jurors but cannot be guaranteed. In fact, a reading of the three jurors' worknotes (Bohart, Tallman, et al., 2011, Appendix 2) reveals wide variations in the use of the 56 criteria and even in their focus on the two key questions of pre-post client change and the role of therapy in client change. In our opinion, this approach significantly reduces the resemblance of this model to the actual experience of jurors within a legal trial. Every jury is presented with two alternative cases to consider and part of their process is to weigh up the plausibility and coherence of the arguments that have been presented.

\section{ISSUES OF PROOF}

\section{Claims and the Burden of Proof}

In both the HSCED and Research Jury models, the central questions to be answered in the adjudication focus on change and causality. This reminds us that both methods have been specifically designed as alternative methods for evaluating the efficacy of therapy. In the PPI model, the range of possible "claims" to be considered by the judges is broader, reflecting Miller's assertion that his approach to the systematic case study "eschews the pursuit of strictly causal hypotheses and theories as goals of clinical research” (2011, p. 7). It is the Advocate in the PPI model who identifies the claims that she believes she can establish in her presentation to the panel (Altman, 2011). In her description of her process in preparing her case, Altman talks about the "reductionistic aspect to the methodology"-that the "case and the treatment components were, in important ways, comprised of more than I was able to reduce to 'propositions'” (p. 72). This is an important point to consider. One of the motivations for investigating single case studies in depth is the richness of the material that 
they offer about the therapeutic experience. Yet to examine every dimension comprehensively would be a challenging task for any researcher-and would require many weeks of commitment from a PPI. Pragmatic decisions must be made and specific claims pursued.

In our opinion, the claims selected by Altman for this PPI were good choices in this first demonstration of the PPI method. Indeed the first two claims - the nature of the client's difficulty and the form of therapy provided — are ones that are not currently examined within the HSCED method but, given its purpose is to evaluate the efficacy of therapy, are important matters to establish. The third claim - that the treatment resulted in increased health and growth-is the central issue of the HSCED and Research Jury models. The fourth claim is the only specific theoretical proposition put forward by the advocate and, in our opinion, has an important place within systematic case studies. We agree with McLeod's (2010) proposal that the potential for generalization from single case studies is likely to be "based on the establishment of cross-case theoretical principles” (p. 28). In fact, current versions of HSCED explicitly seek answers to the questions about possible mediator and moderator variables, on the assumption that our ability to generalize results to other cases will best be based on the presence of causally important client background factors (moderator variables) and intervening change processes (mediator variables).

That it is the Advocate in the PPI model who prepares the claims to be brought before the panel reflects the norm within the legal system that it is the person who brings the claim who carries the "burden of proof.” Miller (2011) provides an explanation of this concept. In the HSCED too we recognize that a burden of proof exists and is carried, in our model, by the affirmative case. The existence of this burden is demonstrated by the order in which the cases are presented to the judges: affirmative brief, skeptic brief, affirmative rebuttal of the skeptic brief, skeptic rebuttal of the affirmative case, affirmative summary narrative, skeptic summary narrative. In this way, the affirmative case, carrying the burden of proof, begins, and the skeptic case, whose role is to test the affirmative's argument, has the final word.

\section{Standard of Proof}

The HSCED method also includes the use of a specific standard of proof, defined as the level of evidence or persuasiveness required to convince a juror or judge to rule in favor of a claim. The two most common and familiar standards of proof in various legal systems are (a) "beyond a reasonable doubt," used in criminal trials, and (b) "preponderance of the evidence," often used in civil trials. In the American legal system, an intermediate standard of proof is used for high stakes civil cases (e.g., child custody cases): "clear and convincing evidence” (see West’s Encyclopedia of American Law quoted by The Gale Group, 2008).

Of these standards of proof, only "preponderance of the evidence" has a clear definition, ie., $>50 \%$ probability. In the Scottish legal system-of relevance to us as a Scot and an American living in Scotland-the standard in civil cases is "on the balance of probabilities," a similar $>50 \%$ probability (Clark, 2009). The highest standard, "beyond a reasonable doubt" depends on the definition of what a "reasonable doubt" is. For example, in the USA, in federal jury cases, this standard of proof is defined as "proof of such a convincing character that a reasonable person would not hesitate to act upon it in the most important of his own affairs” (West's Encyclopedia of American Law, 2008). In other words, something that is "beyond a reasonable doubt" can be treated as though it were 
virtually certain. The equivalent standard of proof in psychology is the $\mathrm{p}<.05$ (i.e., $>95 \%$ probability) standard used in statistical significance testing (Elliott, 2002).

In HSCED we find neither standard of proof to be useful: "beyond a reasonable doubt” is too stringent to be useful for clinical decision-making at a case-by-case study; while "preponderance of the evidence" seems too lenient. For this reason, we have opted for an intermediate standard, equivalent to "clear and convincing evidence," which we have defined as $>80 \%$ probability.

Bohart's Research Jury approach does not address standard of proof, while Miller's PPI method does so unsystematically and qualitatively. For example, in the Miller project, three of the Advocate's four claims (Altman, 2011) are couched in implicit probabilistic language: "without reservation" (claim 1); "it is clear" (claim 2); and "highly probable" (claim 3). Miller et al. (2011) report that the Panel of Psychological Inquiry used the "preponderance of the evidence" standard in their judgment of claims 1, 2 and 3, but omit mention of standard of evidence for claim 4. In addition, they characterize the therapist's testimony as "compelling and convincing” (p. 98). Thus, the general standard of proof here appears to have been the lower standard of "preponderance of the evidence," which we would define in probability terms as $>50 \%$. As noted, in the HSCED method we favor use of the $>80 \%$ standard We feel that the issue of standard of proof within adjudicated case study research is fundamental to the development of this method, and we propose that this concept should be discussed more widely and made explicit in particular studies.

\section{THE ADJUDICATION PROCESS}

We are strongly in agreement with Bohart, Tallman, et al.’s (2011) observation, “All science is ultimately adjudicational at heart” (p. 107) and find their argument about the ubiquitous presence of adjudication within existing scientific processes, such as in journal peer review, highly convincing. We see as very positive and promising the attempts by Miller and Bohart and their colleagues to develop systematic procedures for deciding knowledge claims in case studies.

In order to learn more about the adjudication process, we would have liked to have had access to the full texts of the judges' opinions in Miller's PPI, as it was not possible to truly appreciate their individual views within the brief summary presented (Miller et al, 2011). In contrast, it is clear that understanding in detail the process of adjudication, and how each juror evaluates the evidence presented, is a specific interest of Bohart and his colleagues. We welcomed the access to the jurors' worknotes provided in their case (Bohart, Tallman et al, 2011) and the invaluable opportunity to see for ourselves the widely varying approaches to the evidence taken by each juror. We found the companion study (Bohart, Berry \& Wicks, 2011), which examined the utility of the criteria for evaluating a case study, fascinating. The findings of this study provide promising partial data about inter-judge agreement in the decision-making process of a jury when evaluating evidence that is presented within a case study. This is an aspect of the HSCED that has not yet been researched in detail and the study presented here provides a simple design that could be replicated in order to learn more about the experience of judges within an HSCED adjudication. We would, however, have welcomed a fuller reporting of the different kinds of agreement and disagreement (i.e., on "Yes" vs "No" judgments) between the two raters, as 
these would have enabled us to calculate the proper chance-corrected reliability statistic (Cohen’s kappa).

\section{Selection of Judges/Jurors}

If we are going to use adjudication methods, on what basis should we recruit and select judges? Obviously, research practice is still developing, and there is quite a bit of room for experimentation. Miller (2011) contacted seven highly experienced clinical or counselling psychologists from the academic and practice communities within his state, as well as a senior professor of law; this is similar to Elliott et al.'s (2009) adjudicated HSCED study, in which three distinguished psychotherapy researchers were recruited, each representing a different theoretical approach. The use of highly experienced clinicians as judges makes a lot of sense but can present practical problems for more general use in the field.

For this reason, HSCED studies have moved away from this approach to something closer to Bohart's Research Jury method, which often includes judges of roughly the same professional status as the researchers or therapists, thus beginning to approximate the traditional concept of a jury of one's peers. Bohart, Tallman et al. (2011) used themselves as jurors (two academic psychologists and a research psychologist) and also summarize two previous research jury studies, one using six graduate or undergraduate students and one using Bohart and a graduate student. Bohart, Berry et al. (2011) used two graduate student jurors. For recent and ongoing HSCED research, we have begun the practice of setting up lists of potential judges, similar to panels of potential jurors, via personal contacts and general calls for judges via various professional organizations. As more researchers come to use the method, we find a community of practice developing around HSCED judging, with researchers contemplating using the method eager to volunteer as an opportunity to learn the method from inside, and judges beginning to develop expertise in this role. It will be very interesting to see how this community of HSCED researchers and judges develops over time.

\section{FINAL COMMENTS AND FUTURE DEVELOPMENTS}

The two adjudicated case studies presented here demonstrate the potential offered by this approach to psychotherapy research and, we believe, complement the work that we have achieved so far with the HSCED method. In our view, each model has taken significant steps forward in developing and testing specific aspects of the adjudication process. In this commentary we have highlighted a number of areas in which we believe further development of these models would be beneficial, including the use of direct/indirect sources of evidence, increasing the range of witnesses/perspectives, and, in the case of Bohart's Research Jury model, ensuring that a critical examination of the evidence takes place.

We have also identified several recommendations for further research that we believe are required to fully establish the adjudicated case study approach as a credible research method. Issues that we believe require attention include: (a) further clarification of the advocate/affirmative and critic/skeptic roles; (b) careful consideration of how best to respect the experience of the client and therapist whose encounter is the subject of the adjudicated case study; (c) development and clarification of explicit and appropriate standards of proof; and (d) further research into the processes by which jurors/judges evaluate the evidence. 
On a final note, we are convinced that there is much more that case study researchers can learn from the concepts and methods applied within legal reasoning and practice. As we know, "the issue of generalizability from a single case remains one of the major obstacles in elevating the single case study in psychotherapy research” (Iwakabe \& Gazzola, 2009, p. 601). We are in the early stages of designing a study in which we will critically evaluate whether the concepts and methods typically used in the development of legal precedent can enhance existing strategies used within psychotherapy research for the development of knowledge from a series of case studies. It is envisaged that this study may take us one step closer to Bromley's (1986) conception of:

a kind of 'case law' [that] provides rules, generalizations and categories which gradually systemize the knowledge (facts and theories) gained from the intensive study of individual cases (p. 2).

\section{REFERENCES}

Altman, A.J. (2011). Subjected to proof: The advocate’s perspective. Pragmatic Case Studies in Psychotherapy, 7(1), Article 4, 64-73. Available: http://hdl.rutgers.edu/1782.1/pcsp_journal

Bohart, A.C., Berry, M., \& Wicks, C. (2011). Developing a systematic framework for utilizing discrete types of qualitative data as therapy research evidence. Pragmatic Case Studies in Psychotherapy, 7(1), Article 9, 145-155. Available: http://hdl.rutgers.edu/1782.1/pcsp_journal

Bohart, A., \& Boyd, G. (1997a, December). A qualitative analysis and study of outcome in psychotherapy. Paper presented as part of a poster on "Clients' construction of the therapy process: A qualitative analysis" (poster was split into two papers). Meeting of the North American Society for Psychotherapy Research, Tucson, AZ.

Bohart, A. C., \& Humphreys, C. (2000, June). A qualitative “adjudicational” model for assessing psychotherapy outcome. Paper presented at the meeting of the International Society for Psychotherapy Research, Chicago, Illinois.

Bohart, A.C., Tallman, K.L., Byock, G. \& Mackrill, T. (2011). The “Research Jury” Method: The application of the jury trial model to evaluating the validity of descriptive and causal statements about psychotherapy process and outcome. Pragmatic Case Studies in Psychotherapy, 7(1), Article 8, 101-144, Available: http://hdl.rutgers.edu/1782.1/pcsp_journal

Bromley, D. B. (1986). The case-study method in psychology and related disciplines. Chichester: John Wiley \& Sons Ltd.

Clark, B. (2009). Scottish Legal System Essentials. Dundee: Dundee University Press.

DiGiorgianni, J. (2011). “Anna” and the Panel of Psychological Inquiry: The critic's perspective. Pragmatic Case Studies in Psychotherapy, 7(1), Article 5, 74-87. Available: http://hdl.rutgers.edu/1782.1/pcsp_journal

Elliott, R. (2001). Hermeneutic single case efficacy design (HSCED): An overview. In K.J. Schneider, J.F.T. Bugental \& J.F. Fraser (eds.), Handbook of Humanistic Psychology (pp315-324), Thousand Oaks, CA: Sage.

Elliott, R. (2002). Hermeneutic Single Case Efficacy Design. Psychotherapy Research, 12, 121. 
Elliott, R., Partyka, R., Alperin, R., Dobrenski, R., Wagner, J., Messer, S., Watson, J.C., \& Castonguay, L.J. (2009). An adjudicated hermeneutic single-case efficacy design study of experiential therapy for panic/phobia. Psychotherapy Research, 19, 543-557.

Elliott, R., Slatick, E., \& Urman, M. (2001). Qualitative change process research on psychotherapy: Alternative strategies. In J. Frommer \& D.L. Rennie (Eds.), Qualitative psychotherapy research: Methods and methodology (pp69-111). Lengerich, Germany: Pabst Science Publishers

Iwakabe, S. \& Gazzola, N. (2009). From single-case studies to practice-based knowledge: aggregating and synthesizing case studies. Psychotherapy Research, 19, 601-611.

Llewelyn, S. (1988). Psychological therapy as viewed by clients and therapists. British Journal of Clinical Psychology, 27, 223-238.

Mackrill, T. (2011). The case of "Jane and Joe": A diary-based, cross-contextual case study. Pragmatic Case Studies in Psychotherapy, 7(1), Article 11, 187-229. Available: http://hdl.rutgers.edu/1782.1/pcsp_journal

MacLeod, R., Elliott, R., \& Rodgers, B. (in press). Process-Experiential/Emotion-focused Therapy for Social Anxiety: A Hermeneutic Single-Case Efficacy Design Study. Psychotherapy Research.

McLeod, J. (2010). Case Study Research in Counselling and Psychotherapy. London: Sage.

Miller, R.B. (2011). Real Clinical Trials $\left(\mathrm{RCT}^{1}\right)$ - Panels of Psychological Inquiry for Transforming Anecdotal Data into Clinical Facts and Validated Judgments: Introduction to a Pilot Test with the Case of “Anna”. Pragmatic Case Studies in Psychotherapy, 7(1), Article 2, 6-36. Available: http://hdl.rutgers.edu/1782.1/pcsp_journal

Miller, R.B., Kessler, M., Bauer, M., Howell, S., Krieling, K., \& Miller, M. (2011). Findings of the Panel of Psychological Inquiry convened at St Michael's College, May 13, 2008: The case of “Anna”. Pragmatic Case Studies in Psychotherapy, 7(1), Article 7, 95-100. Available: http://hdl.rutgers.edu/1782.1/pcsp_journal

Podetz, S.M. (2011a). Seeing beyond the scars: A testament to Anna. Pragmatic Case Studies in Psychotherapy, 7(1), Article 3, 37-63. Available: http://hdl.rutgers.edu/1782.1/pcsp_journal

Podetz. S.M. (2011b). Panel of Psychological Inquiry: The therapist's perspective. Pragmatic Case Studies in Psychotherapy, 7(1), Article 6, 88-94. Available: http://hdl.rutgers.edu/1782.1/pcsp_journal

Stephen, S., Elliott, R., \& MacLeod, R. (2011). Person-centred therapy with a client experiencing social anxiety difficulties: a hermeneutic single case efficacy design. Counselling and Psychotherapy Research,11(1), 55-66.

The Gale Group (2008). West's encyclopedia of American law ( $2^{\text {nd }}$ Ed). Online document: http://legal-dictionary.thefreedictionary.com/Standard+of+proof 